\title{
Analysis of physical human-robot interaction for motor learning with physical help
}

\author{
Shuhei Ikemoto ${ }^{\mathrm{a}, \mathrm{b}^{*}}$, Takashi Minato ${ }^{\mathrm{b}}$ and Hiroshi Ishiguro ${ }^{\mathrm{a}, \mathrm{b}}$ \\ ${ }^{a}$ Adaptive Machine System, Osaka University, Osaka, Japan; ${ }^{b}$ Japan Science and Technology Agency, ERATO, Asada Synergistic \\ Intelligence Project, Saitama, Japan
}

(Received 29 September 2008; final version received 10 February 2009)

\begin{abstract}
In this paper, we investigate physical human-robot interaction (PHRI) as an important extension of traditional HRI research. The aim of this research is to develop a motor learning system that uses physical help from a human helper. We first propose a new control system that takes advantage of inherent joint flexibility. This control system is applied on a new humanoid robot called $\mathrm{CB}^{2}$. In order to clarify the difference between successful and unsuccessful interaction, we conduct an experiment where a human subject has to help the $\mathrm{CB}^{2}$ robot in its rising-up motion. We then develop a new measure that demonstrates the difference between smooth and non-smooth physical interactions. An analysis of the experiment's data, based on the introduced measure, shows significant differences between experts and beginners in human-robot interaction.
\end{abstract}

Keywords: physical human-robot interaction; humanoid robot; pneumatic actuator; rising-up motion

\section{Introduction}

In recent years, many researchers have been focusing on generating robot motions by learning. However, it is difficult to generate real robot motion due to the noise in real environment or breakdowns of the robot's hardware during the many trials required for learning. In particular, motor learning for humanoid robots is very difficult because a humanoid robot normally has complex dynamics. Therefore, the tasks have been limited to simple motions or simulations have been applied in order to avoid the risk of breakdown. In this research, we consider a scheme in which a human can physically help the robot's motor learning in order to reduce the risk and the number of trials in the learning.

In order to realise this kind of learning scheme, the class of Human-Robot Interaction (HRI) where a human and a robot are influencing each other by physical contacts has to be considered. So far, many researchers have been focusing on HRI in order to realise communication between a human and a robot. However, physical interaction has not been considered much because it is very difficult to realise. Therefore, the requirements in terms of hardware and software to realise HRI with physical contacts have also not been clarified. Moreover, an analysis method for the physical interaction has not been established because the interactions considered in the existing studies have been focusing on symbolic communications that usually assume turn-taking. Furthermore, it is clearly different from teaching and playback (Asada and Asari 1988) which has been focused on in the domain of control engineering. In this paper, we study this type of HRI in order to clarify the requirements of robot hardware and software by implementing an example behaviour and analyse the realised behaviour in order to figure out how to evaluate physical interaction behaviours. Hereafter, we denote the class of HRI considered in this research as physical human-robot interaction (PHRI).

In human motor learning there are several instances where a teacher physically supports a learner to enable him or her to accomplish a certain task or motion. For example, babies often receive physical help from their parents in order to learn to walk or stand. This kind of physical assistance is not limited to the early years, but occurs at all ages. To learn from this kind of physical interaction, a robot needs to move appropriately to the force applied by the human helper. This suggests that even if the robot is actively moving, the motion has to be changed by the human's physical help because the robot has no a priori knowledge of the human's intention or motion. In order to enable such a passive reaction, the robot's joints have to have a high flexibility in response to the applied force, which also ensures higher safety for both the robot and the human teacher. We assume that a flexible-joint robot, such as a robot actuated by pneumatic actuators, is suitable for PHRI.

In this research, we propose a control system that takes advantage of flexible joints and that can exploit PHRI for motor learning problems. The proposed control system is based on the switching of a few desired postures in a feedback control system. The advantages of this control system are shown by implementing a specific behaviour belonging

${ }^{*}$ Corresponding author. Email: shuhei.ikemoto@ams.eng.osaka-u.ac.jp 
to the class focused in this research, in which the robot stands up by relying on physical help from a human. To investigate a learning system that employs such physical help from a human, we have to know how to evaluate the interactions. In order to clarify which features need to be considered by the learning systems, we also analysed the data taken in the rising-up motion to quantify the difference between smooth and non-smooth physical interaction. The results of this analysis helped us to better understand PHRI. In particular, we devised one factor that is assumed necessary for motor learning with physical help.

\section{Related work}

In recent years, research on HRI has become particularly interesting. In most studies on HRI (e.g. Kanda et al. 2002; Suga et al. 2004; Noda et al.), the HRI has been symbolised by a set of meaningful motions described in advance. However, the symbolisation approach is not suitable for PHRI because the robot's motions are generated through the interaction.

So far, safeness and dependability of a robot's hardware and control system have been emphasised in order to put the robot into practical use (Alami et al. 2006). In this research, we focus on the case where a robot works with physical help from a human and propose a control system and a learning system that makes use of this physical help. In detail, the robot needs to work both passively and actively in accordance with the applied force of the human helper. Here, passive motion refers to a motion generated by a force according to the mechanical impedance of its own joints. In addition, this type of motion does not apply force to the human. Active motion refers to motion generated by activating each joint. The resultant motion is influenced by the applied force of the human helper. The purpose is to apply a force to the human in order to elicit a reaction that helps to achieve the goal of the given task. In PHRI, both a passive-motion-dominant phase and an active-motion-dominant phase generally appear during the interaction. In this paper, we focus on motions that can be separated into active and passive motions. This helps us to draw clearer conclusions about the results of our research.

Kosuge et al. (2000) developed a robot that could convey an object with a human's help by implementing a passive motion. In this research, the robot's passive motion is generated by emulated impedance defined by the change in the robot's locomotion and posture according to this impedance. However, the control system proposed in (Kosuge et al. 2000) is not adequate for the task we focus on, since we need the robot to perform both passive and active motions.

In addition, Kosuge et al. (2003) developed a robot that could dance with a human by adaptively changing the robot's dance steps according to the applied force. In this second work, the passive motion is implemented by an emulated impedance defined as above and the active motion is implemented as independently scheduled dance steps of the robot. However, the robot has to know how to move to achieve the goal by itself before starting a physical interaction with the human. Therefore, physical interaction can be expressed as an advanced motor skill of moving by itself. This assumption is difficult to achieve in learning scenarios because a robot learning a motor skill from physical interaction does not have a priori knowledge of the target motor skill. Therefore, this control scheme is not suitable for our research. Most importantly, we need a control system that allows physical interaction with a human, even before any motor learning has started.

In this research, we realised the robot's passive motion as a fundamental motion by using the inherent flexibility of the robot's joints, which are driven by pneumatic actuators. We then proposed a control system that is suitable for PHRI by exploiting the flexibility of the joints. Additionally, we analysed the realised PHRI in order to determine a measure suitable for developing a learning system that uses PHRI for learning.

\section{3. $\mathrm{CB}^{2}$ : A child robot with biomimetic body}

\subsection{Hardware of $\mathrm{CB}^{2}$}

In most related research, robot joints are driven by electric motors with decelerators. Therefore, the robot's joints need to be controlled by impedance control that emulates mechanical impedance in order to ensure the safety of PHRI and generate the passive motions. At the same time, the robot needs torque sensors for each joint, as well as calculation capability for emulating the joint's impedance. On the contrary, robots with inherent high flexibility in their actuators do not need sensors or special control to ensure safety or the generation of passive motion. Therefore, using a robot with flexible actuators is more suitable for research on physical interaction.

Figure 1 shows the robot $\mathrm{CB}^{2}$ (Minato et al. 2007) used in this research. The $\mathrm{CB}^{2}$ robot has the following features:

- It is $130 \mathrm{~cm}$ high and weighs about $33 \mathrm{~kg}$.

- It has 56 degrees of freedom (DOFs).

- All joints, apart from the joints used to move the eyes and eyelids, are driven by pneumatic actuators.

- All joints, apart from the joints used to move the fingers, have potentiometers.

- The whole body is covered in soft silicone skin.

- It has 197 PVDF tactile sensors throughout its body.

- It has a camera in each eye and a microphone in each ear.

The joints have low mechanical impedance due to the compressibility of air. The joints can also be made completely passive by the system discontinuing the air 

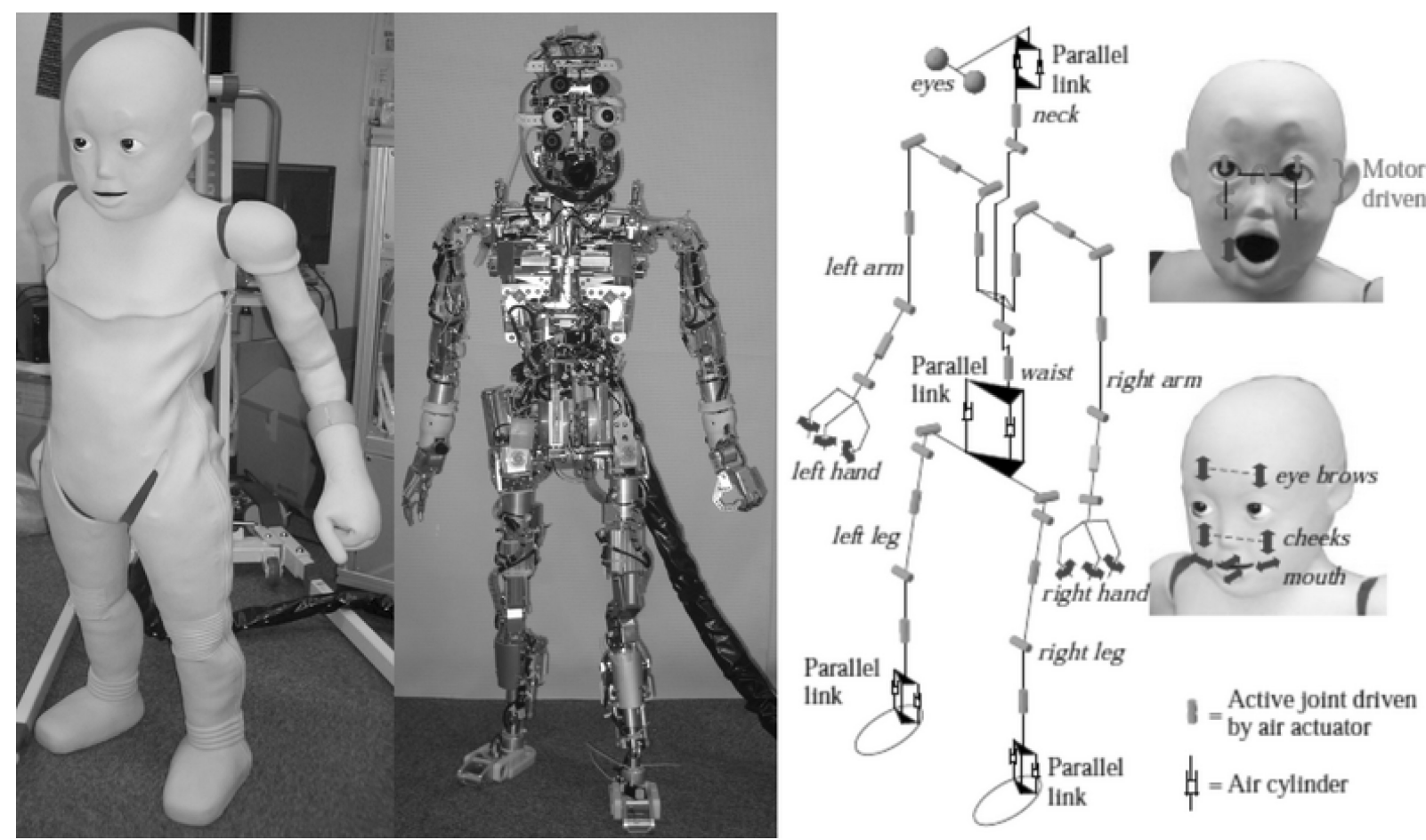

Figure 1. $\mathrm{CB}^{2}$ (Robot actuated by pneumatic actuators).

compression during the motion. This helps the robot to perform passive motion in physical interaction and helps to ensure the human helper's safety. However, the pneumatic actuators are very difficult to control. For example, precise trajectory tracking is difficult to implement because of the inherent strong nonlinearity of pneumatic actuators. However, this is not a significant problem in our research, since we emphasise the physical interaction between humans and robots rather than the robot's precise autonomous control.

\subsection{Control System of $\mathrm{CB}^{2}$}

The pneumatic actuators allow passive motion in physical interaction without emulating mechanical impedance. As a result, the control system becomes simpler for PHRI than the control systems used in traditional robot systems. Moreover, even if the input signal changes drastically, the flexible joints will generate smooth-looking motion. This is facilitated by the air's compressibility working as a lowpass filter. Based on these advantages, we propose a control system that is suited for human-robot physical interaction. The idea of this proposed control system is to implement $\mathrm{CB}^{2}$ 's active and passive motions as movements generated by drastic changes in posture and adaptive motions generated accordingly by external force and the flexibility of the joints. In this control system, the dynamics of $\mathrm{CB}^{2}$ strongly influence the generation of both of active and passive mo- tions. Additionally, this indicates the decay of control accuracy. However, it is not a significant problem in the situations where a human counterpart influences and changes the robot's motions because it is impossible to schedule the robot's motion in advance.

Figure 2 shows the proposed control system implemented for $\mathrm{CB}^{2}$. In this figure, $t$ refers to time, $\boldsymbol{x}$ is the currently realised posture vector, $\boldsymbol{x}^{*}$ is the desired posture vector, and $\boldsymbol{x}^{*(i)}(i=1,2, \ldots, n)$ is a set of $\boldsymbol{x}^{*}$. For instance, an angular value can be used for an element of $\boldsymbol{x}$. Each angular value of the joints is controlled by PD-control which is a feedback control that uses the error of the angular value and its derivative. The motion is generated by selecting and switching the desired posture vector several times in the control system.

In this system, drastic switching of the desired posture vector leads to the generation of large drive torques in each joint, which means performing an active motion applies force to the human helper. Therefore, switching to the desired posture corresponds to a change in the robot's dominant motion from passive to active motion. As the robot's posture approaches the desired posture, the passive motion gradually becomes the dominant motion of the robot. In this phase, the parameters (desired posture vector, switching timing and feedback gain) strongly affect both active and passive motions. Therefore, in the proposed control system, we must choose appropriate values for these three parameters. 


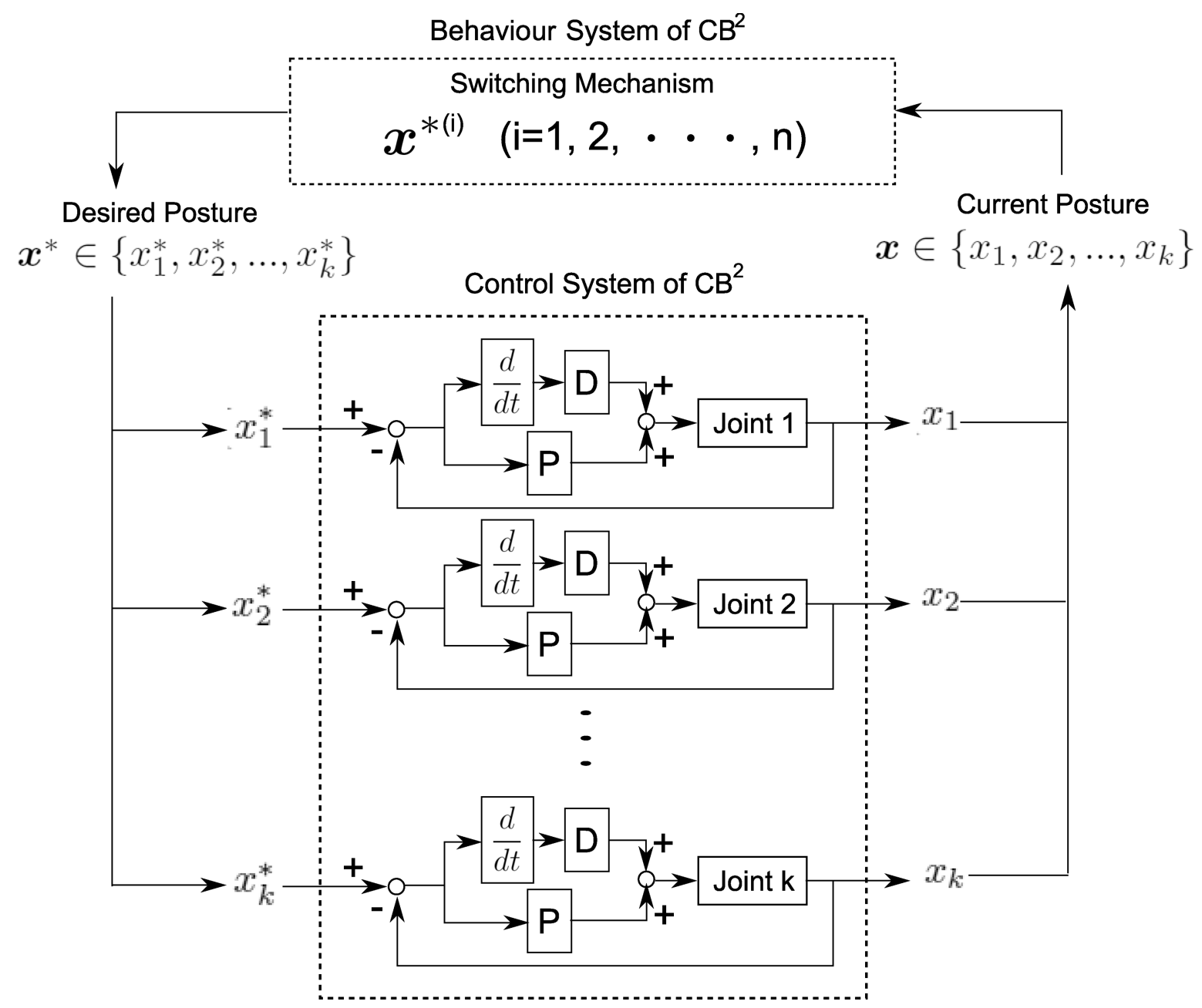

Figure 2. Control system for rising-up behaviour.

\subsection{Implementation of rising-up behaviour}

In this research, we focus on the act of physical interaction where a human has to help a robot in its rising-up behaviour. To realise this interaction, we determined the above parameters according to the following procedure:

1. configure the initial posture

2. configure the final posture and the feedback gain that can be assumed to maintain a standing posture

3. configure the intermediate postures that can be assumed to lead to the final posture from the initial posture

4. configure the timing for switching of each desired posture that can make the robot stand while receiving physical help.

The switching times were defined as conditional expressions using previously realised posture vectors and the currently desired posture vector in order to decide whether to switch the desired posture vector. For example, the start of the robot's motion is decided when the robot posture changes due to an applied force from the human helper. This indicates that the robot motion is adaptively generated by the interaction.

Figure 3 shows the frame format of the realised risingup behaviour. As indicated by the figure, we assume that the robot can realise the rising-up behaviour by switching the desired posture vector only two times. However, the system needs a trial-and-error process to determine the appropriate switching rule that can realise a successful rising-up motion. It is nearly impossible to realise a similar interaction using traditional robots and control systems. Therefore, the proposed system seems better suited for such physical interaction.

In Section 4, we explain a proposed evaluation method for PHRI, experiment conducted and the results that were gathered. 

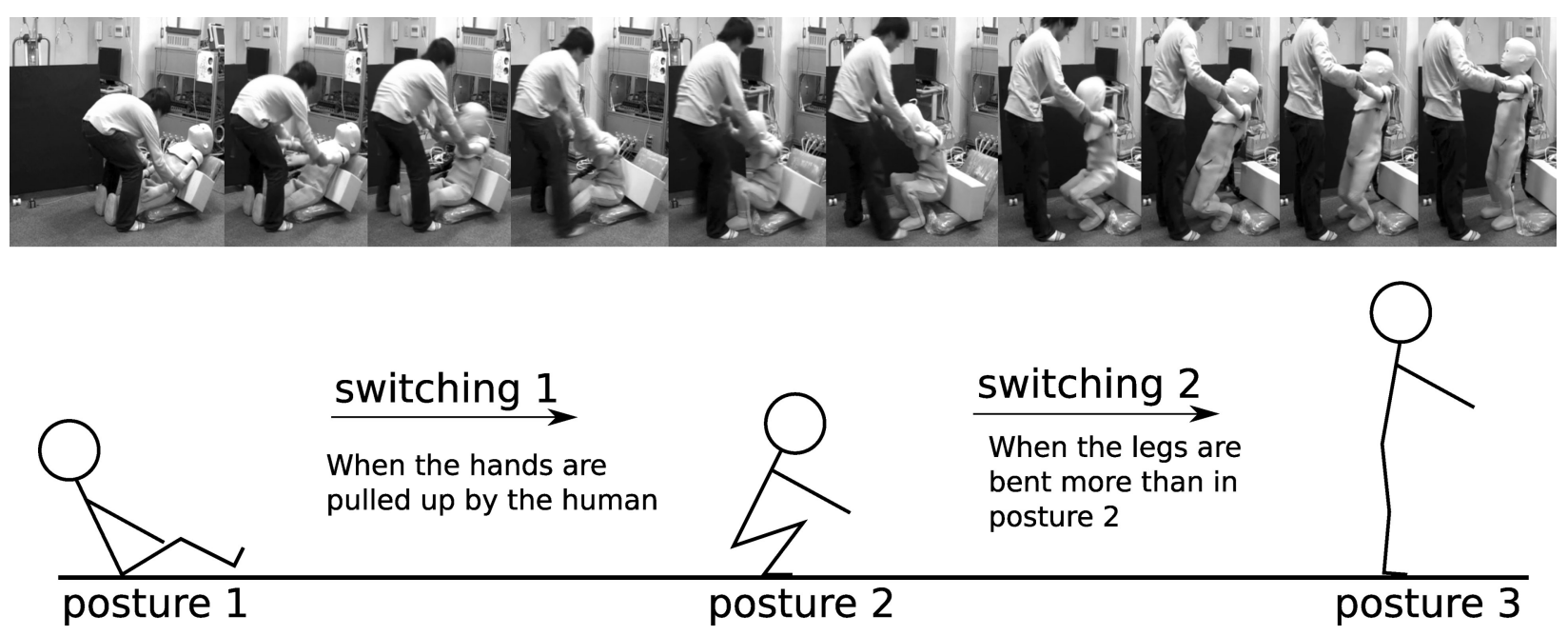

Figure 3. Realised physical human-robot interaction.

\section{Experiment}

\subsection{Experimental setup}

An evaluation of physical interactions must be made before we investigate a learning system operating through such physical interactions. In order to clarify the difference between successful and unsuccessful interaction, we conducted an experiment focusing on rising-up behaviour.

In this experiment, we classified interactions according to their success into 'smooth', 'non-smooth' and 'failed':

\section{- Smooth}

If the robot could stand up with subjectively smooth interaction, it is classified as 'smooth'. We denote only this kind of interactions 'successful'.

\section{- Non-smooth}

If the robot could stand up without subjectively smooth interaction, it is classified as 'non-smooth'. For example, when the subjects used their legs to rise up the robot or the robot's knees touched on the ground, the interactions are classified into 'non-smooth'.

\section{- Failed}

If the robot could not stand up, the interaction is classified as 'failed'.

During the rising-up motion, we recorded the subject's motion by using a motion capture system. The motion capture markers were located so as to record the data of the upper body motion. Two markers were placed on the elbow, one on the shoulder, one on the hand, one on the back, and two on the waist. The system's sampling frequency was $60 \mathrm{~Hz}$. For recording the robot's motion data, we used the potentiometer in each joint with a sampling frequency of $100 \mathrm{~Hz}$. During the experiment, the data was stored in a database with a time-stamp that was synchronised between both systems.
Two subjects participated in the experiment. One of them was an expert who knew the robot's motion in detail and could perform smooth rising-up motions. The other subject was a beginner who didn't know much about the robot's motion. Before performing the interaction, they were instructed as to how each robot would stand up in place by means of a video of a successful interaction. They performed five trials. We compared successful trials with failed trials to clarify the difference between smooth and non-smooth physical interaction.

\subsection{Analysis of interaction}

Abe et al. (2003) showed the difference in motor skills between an expert and a beginner of ceramic art by focusing on the cross-correlation values of the time-series of posture data taken using a motion capture system. Inspired by their study, we focused on the cross-correlation value between the subject's and the robot's motion data to see the difference between successful and unsuccessful interaction. However, it is difficult to apply this analysis method directly to the rising-up motion because it is non-periodical and therefore it is not suitable to be evaluated by cross-correlation values of the whole data area. In this paper, we employ the method to a local data area in order to calculate a cross-correlation value which can visualise the relationship between the subject and the robot motion data each time. In particular, we focus on the relationship between the amounts that the subject's motion and the robot's motion change. In the following, we explain how to calculate the amount of motion change and how to calculate cross-correlation value.

\subsubsection{Calculation of posture-change norm}

The subjects' motion data from the motion capture system were recorded in the form of marker positions in an 

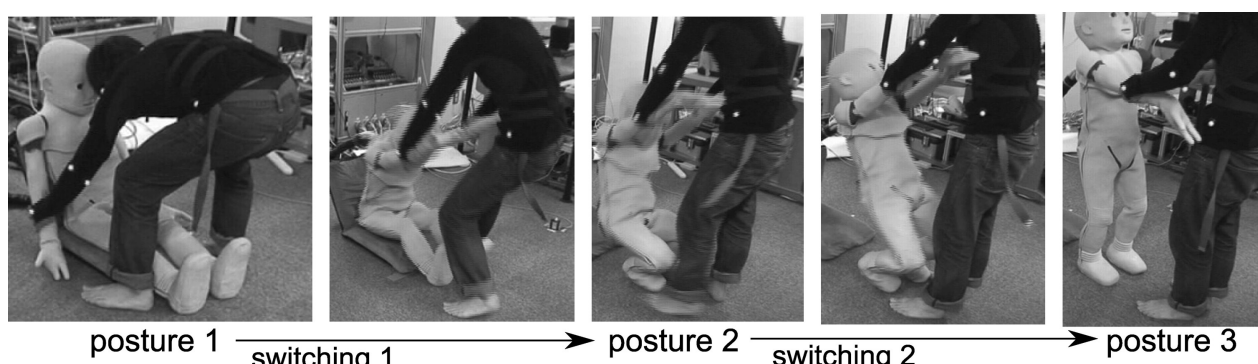

posture 1 switching 1
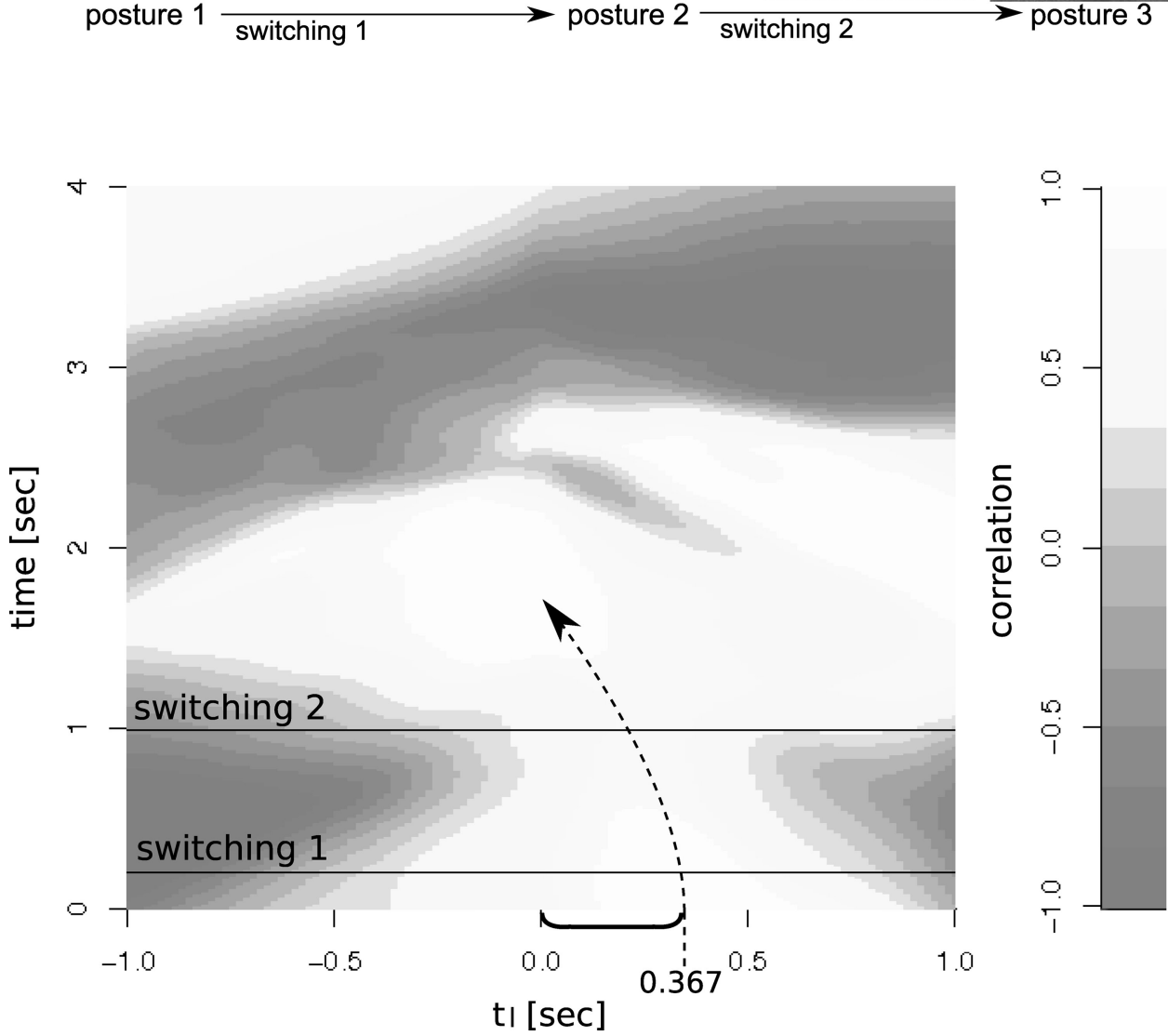

Figure 4. Result of smooth interaction by an expert.

absolute coordinate system. The robot motion data were taken from potentiometers and represent angular values of the robot joints. Both of these motion data are time-series data of realised posture in each time. However, they have to be transferred into a comparable representation because their dimensions are different from each other and absolute positions of marker and angular values are difficult to compare.

We employed the posture change norm, which measures the amount of motion change, in order to calculate crosscorrelation values. The norms of the subject and the robot were calculated using the following method:

1. Calculate the robot's posture-change norm, $a$, according to the Euclidean distance between the data of $t$ and $t-1$ in the posture space $\boldsymbol{X}$ defined by using each joint angle as a base. The head, neck and hand joint angles were not used as bases because these joints were not controlled in the experiment.

$$
a_{(t)}=\left\|\boldsymbol{x}_{(t)}-\boldsymbol{x}_{(t-1)}\right\|_{2}, \quad \boldsymbol{x} \in \boldsymbol{X} .
$$

2. Calculate the subject's posture-change norm, $b$, according to the Euclidean distance between the data of $t$ and $t-1$ in the posture space $\boldsymbol{Y}$ defined by using each marker's position as a base. The markers on the elbow were ignored because they contained too much noise.

$$
b_{(t)}=\left\|\boldsymbol{y}_{(t)}-\boldsymbol{y}_{(t-1)}\right\|_{2}, \quad \boldsymbol{y} \in \boldsymbol{Y} .
$$



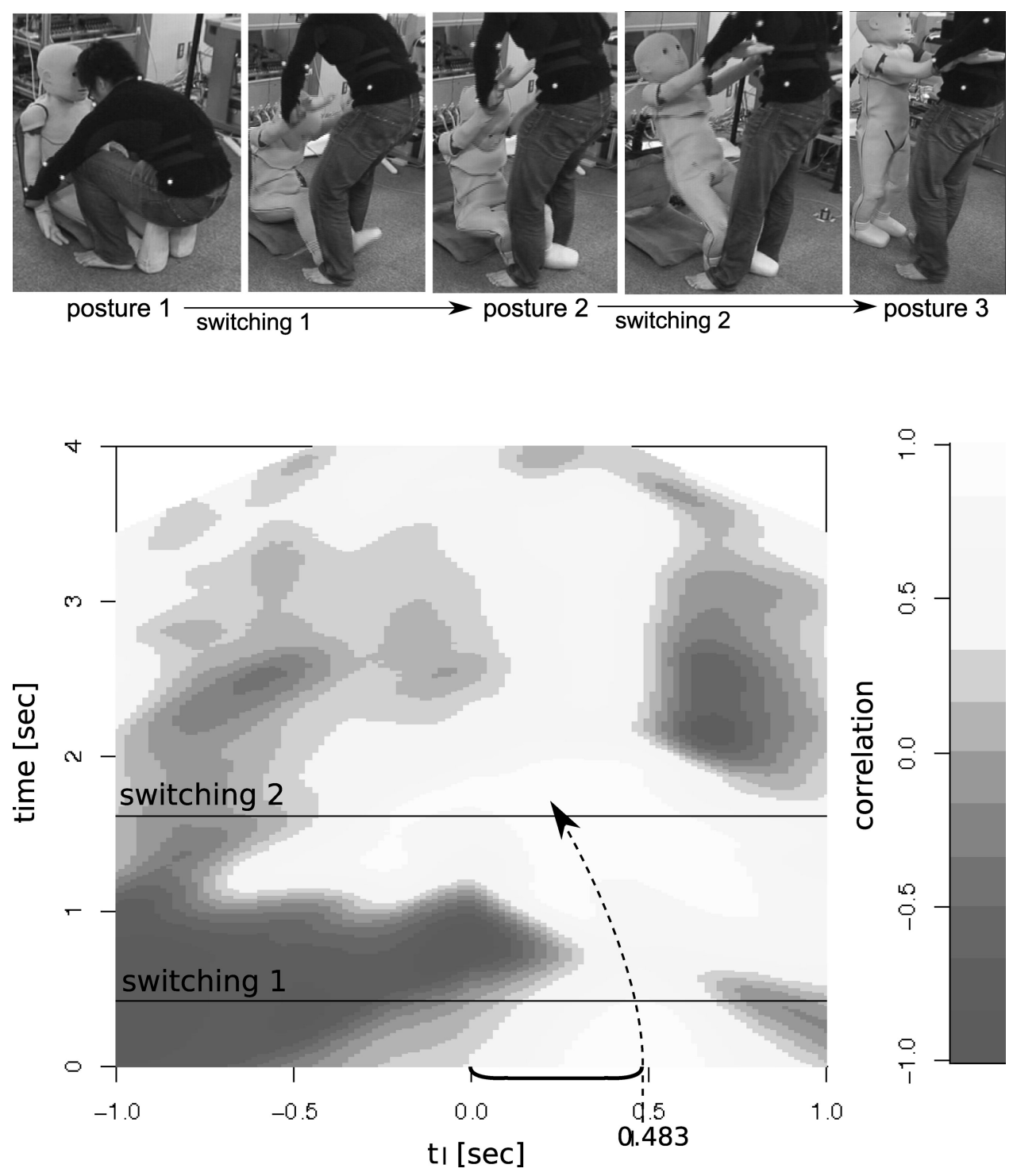

Figure 5. Result of smooth interaction by a beginner.

The posture-change norms $a_{(t)}$ and $b_{(t)}$ are always positive scalar values because they directly indicate the $\mathrm{Eu}-$ clidean norm in the robot's posture space and the subject's posture space, respectively. Here, the values indicate the amount of motion change which means how much the posture was changed. However, these values do not contain information about how the posture was changed. In Section 4.2.2, we calculate the cross-correlation values by using $a_{(t)}$ and $b_{(t)}$ under several conditions.

\subsubsection{Cross-correlation value for physical interaction}

Abe et al. (2003) recorded the subject's motion data by a motion capture system and employed cross-correlation values calculated between each couple of marker position changes in order to clarify the difference in motor skills between an expert and a beginner of ceramic art. We think the research presented in this paper would be helpful for the exploitation of cross-correlation to understand the features of the rising-up motion, because it is important to consider the joint dynamics of the subject and the robot in the interaction as if it were one person's motion. However, it would be difficult to understand the features of the rising-up motion if the cross-correlation values were calculated from whole time-series dataset in the interaction which is a non-periodic motion. Moreover, the robot behaves both actively and passively due to the proposed control system explained in Section 3.2 and the subject also behaves both actively and passively according to the robot behaviour. 

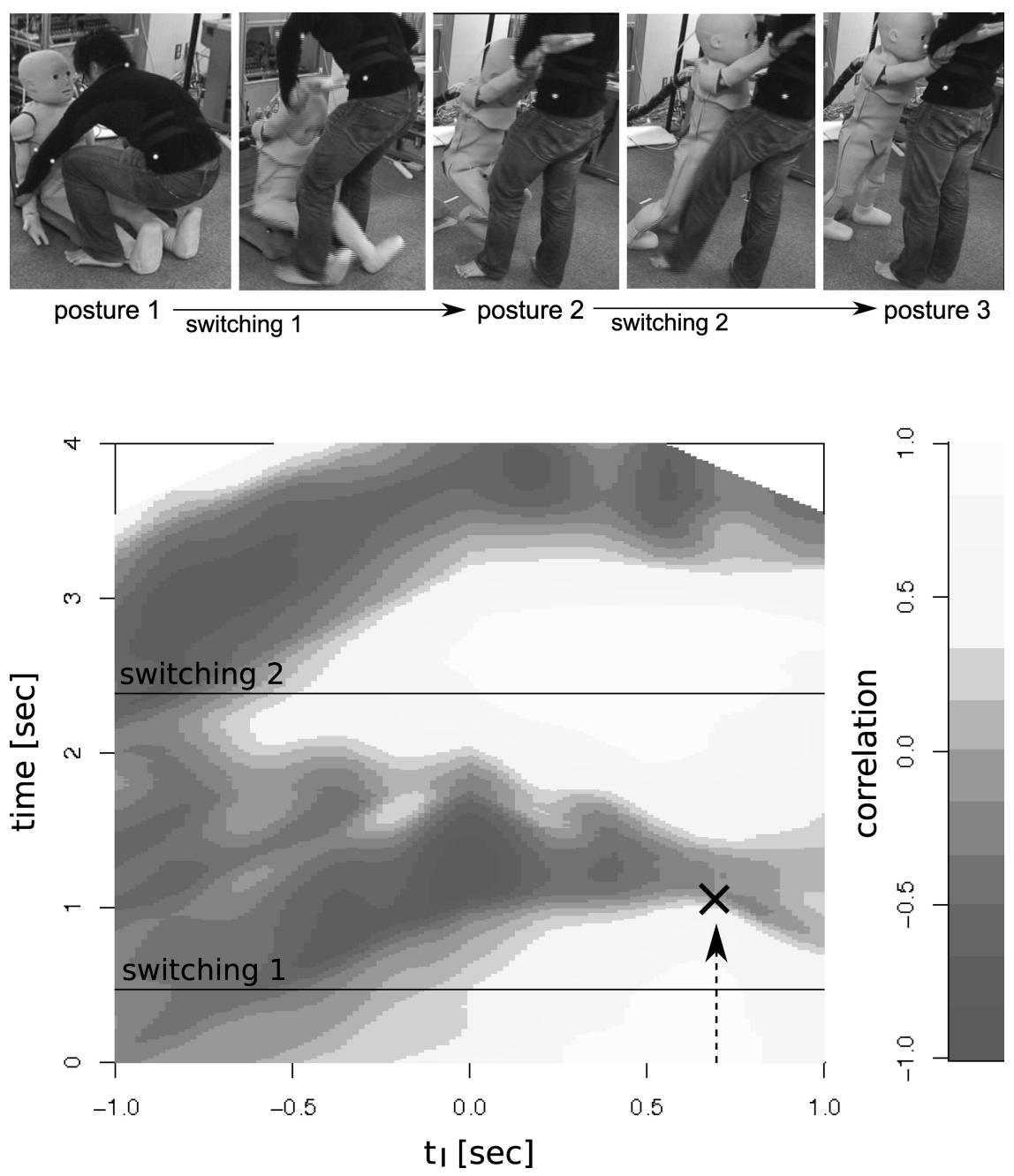

Figure 6. Result of non-smooth interaction by a beginner.

In this paper, we employ local data areas in the datasets to investigate the process of the interaction. In other words, we calculated the cross-correlation values between two time windows of the same length over the robot's data $\left\{\boldsymbol{x}_{(t)} \mid t=\right.$ $\left.t_{r} \ldots t_{r}+t_{n}\right\}$ and the human's data $\left\{\boldsymbol{y}_{(t)} \mid t=t_{h} \ldots t_{h}+t_{n}\right\}$, where $t_{r} \geq 0$ and $t_{h} \geq 0$ are, respectively, the start time of the window over robot's data and over human's data.

The posture-change norms of the subject and the robot correspond to the rate of posture change. Therefore, if there was a strong cross-correlation between the posture-change norms of the subject and the robot, the postures would appear to change synchronously. In Section 3, we explained that the start of the robot's motion is decided when the robot's posture is changed due to the force applied by the subject. Therefore, there is a slight shift between the subject's and robot's motions. This time-delay will decrease as the active motion becomes dominant.

In terms of the delay at the start time, we calculate the cross-correlation value for different values of the parameter $t_{l}$ defined as $t_{l}=t_{r}-t_{h}$, which indicates the temporal difference of the data areas where $t_{r}$ and $t_{h}$ are positive values.

\subsection{Results of analysis}

Figures 4-7 depict the analysis results and the sequential photographs of smooth interaction by the expert, smooth interaction by the beginner, non-smooth interaction by the beginner and failed interaction by the beginner. Even the failed interaction indicates that the subject could lead the robot in standing up. We then subjectively judged whether the interaction was smooth. In these figures, the vertical axis and the horizontal axis indicate $t$ and $t_{l}$, respectively, the amplitude of the cross-correlation value is expressed by the colour, $t=0$ indicates when the subject grasped the hands of the robot and pulled it up. The 'switching 1' and 'switching 2' lines indicate when the desired posture vector was switched in each trial. Here, the duration of data area $t_{n}$ was appropriately configured as $t_{n}=1(\mathrm{sec})$ by trial-and-error. 

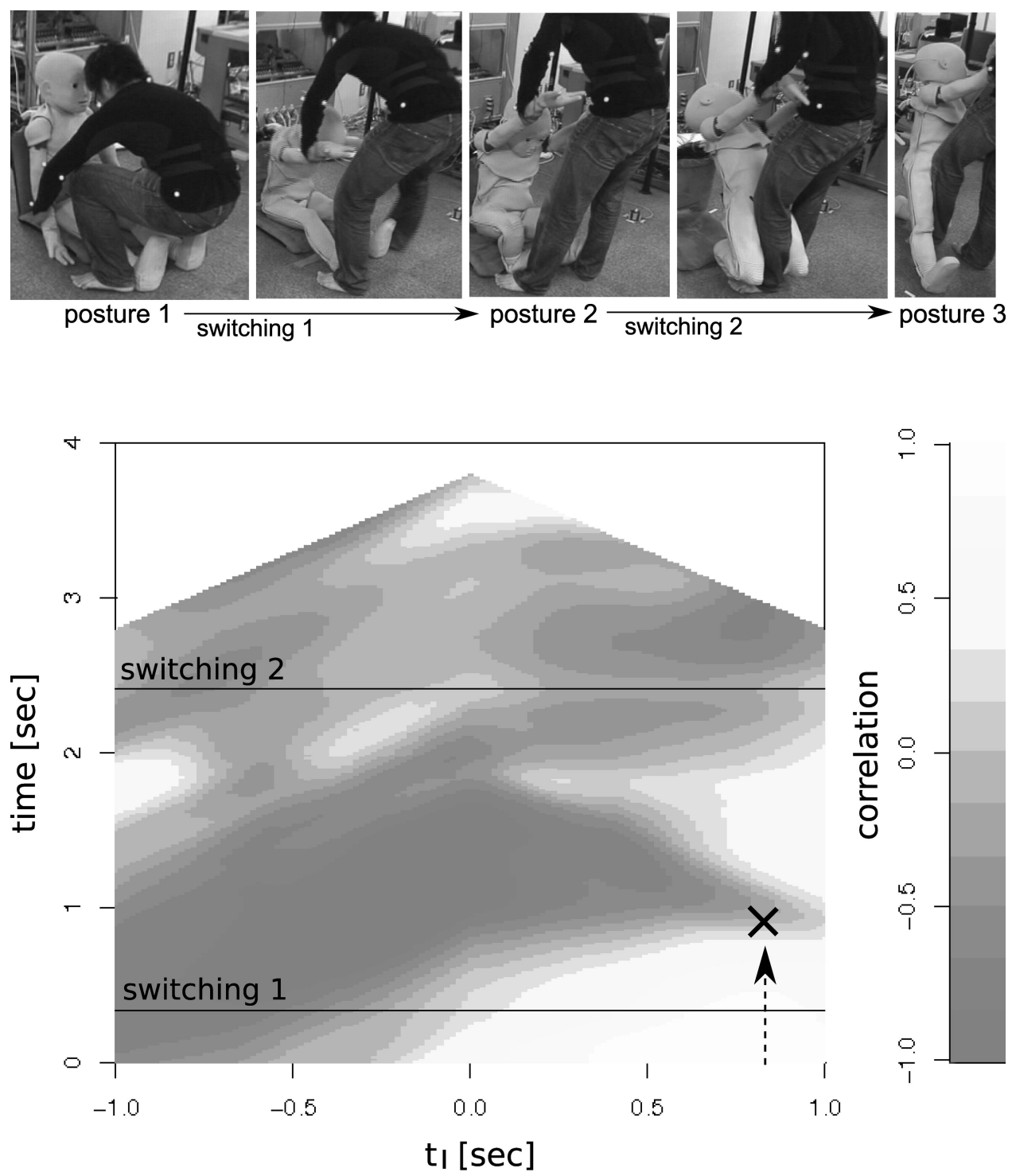

Figure 7. Result of failed interaction by a beginner.

In detail we choose a value such that the plots of failure trials and smooth trials were easily distinguishable. The parameter $t_{l}$ was changed such that $-1 \leq t_{l} \leq 1$, because the robot normally starts the motion no longer than 1 second later.

In all the results, we see a strongly positive correlation in the area $t_{l}>0$ at the beginning of the interaction. This means that the beginning of the robot's posture change was led by the subject's posture change based on the configured parameters as previously explained. In the result of the expert's trial, we can see that the maximum correlation is located at a smaller value of the parameter $t_{l}$ than in the beginner's trial $(0.367$ for the expert successful trial and 0.483 for the beginner's trial, respectively). This indicates that the expert could lead the start of the robot's motion smoothly with less instruction because he understood the robot's motion in greater detail than did the beginner.

In the smooth interaction's result, we can see that the positive correlation value had been maintained during the interaction (dashed lines). On the other hand, in the results of non-smooth and failed interactions, we cannot see a continuous positive correlation. This indicates that smooth interaction needs the human helper to follow the robot's motion by gradually changing his own posture until the posture change is synchronised with the robot's posture change. This is apparent because the strongly positive value appears at $t_{l} \approx 0$ after switching 2 .

From these results, we conclude that to perform smooth interaction requires that the posture variations have to be gradually synchronised in the interaction. If the interaction 
was non-smooth or failed, the continuous positive correlation and this process would not appear. The expert user achieved better results than the beginner, so the key to successful physical interaction seems to be an understanding of the robot's motion and dynamics. The results were obtained from several trials by the expert and the beginner. Moreover, the physical interaction performed in the experiment is limited to the rising-up behaviour. Therefore, these results should be verified by testing other physical interactions and with a larger number of subjects. Although further analysis is required, it is difficult to maintain the reliability of the analysis because compressing the data taken from the motion capture system and the potentiometers into the posture-change norms leads to a loss in the granularity of the data. However, this is still a promising approach to qualitatively distinguish between smooth and non-smooth interactions. In order to use physical help from a human in motor learning, it is necessary to evaluate whether the robot's motion can lead to a meaningful physical interaction that improves the motion. We assume that the results can be used to qualitatively distinguish whether the robot's motion could lead to a successful interaction. In the next section, we discuss which factor can be used to improve the motion.

\subsection{Comparison of desired posture switching times}

In the set of control parameters used in our system, the switching time is the only parameter that is decided based on sensor information taken during the interaction. Therefore, here we discuss the importance of this parameter using the results from the last experiment.

Table 1 shows the applied switching times of the four trials shown in Figures 4-7. We can see that the smooth interaction has a shorter temporal difference between switching 1 and switching 2 than the non-smooth and failed interactions. This indicates that the subject was required to lead the robot to switching 2 quickly from switching 1 by applying a force to help the robot's dynamics in the stand-up motion.

In order to confirm this hypothesis, we conducted a second experiment. Six subjects participated in this trial. They were instructed as in the above-described experiment. All of them were beginners who did not know much about the robot's motion. They each performed the rising-up motion five times. We compared the applied switching times of successful trials with failed and non-smooth trials. The

Table 1. Switching times in each trial (sec).

\begin{tabular}{lcc}
\hline Result & Switching 1 & Switching 2 \\
\hline Expert (smooth) & 0.19 & 0.98 \\
Beginner (smooth) & 0.41 & 1.60 \\
Non-smooth & 0.46 & 2.37 \\
Failed & 0.32 & 2.4 \\
\hline
\end{tabular}

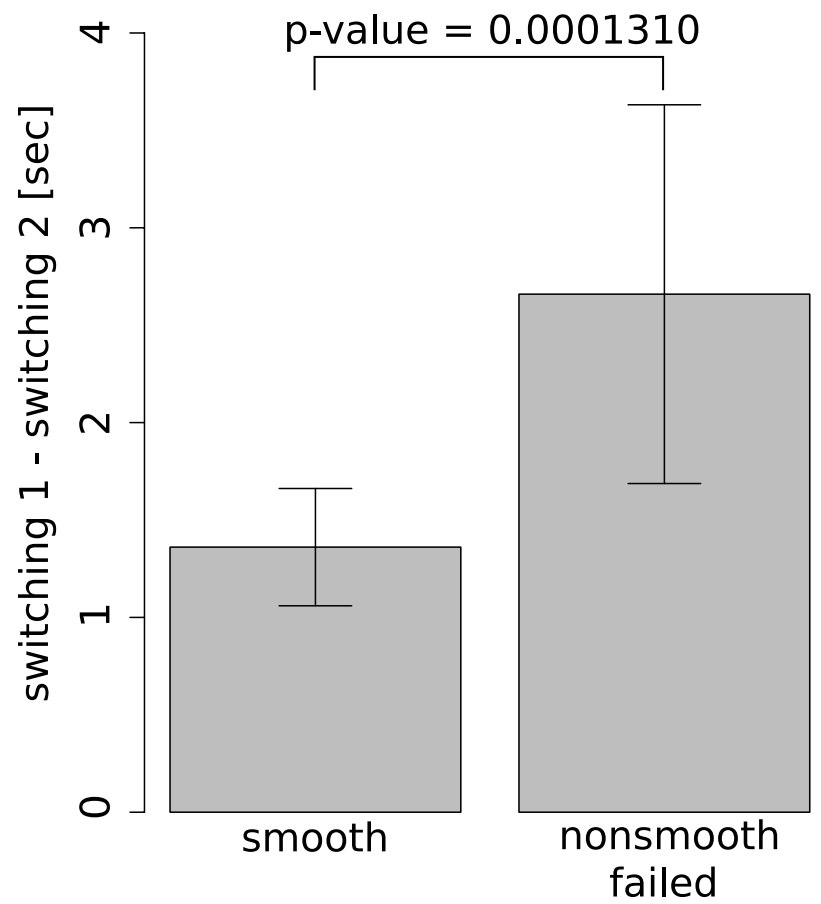

Figure 8. Result of statistical test of switching timings.

number of data is 15 for each category. Figure 8 shows the averages and the variances of successful and unsuccessful trials. From the result of $t$-test, we can confirm our hypothesis that smooth interaction has a shorter temporal difference between switching 1 and switching 2 than non-smooth and failed interactions significantly. Therefore, if this switching rule could adapt to the applied force of the human helper, the resulting motion would always lead to a smooth interaction. This shows the possibility of modifying the switching rule to improve the robot's motion.

\section{Discussion and future work}

In the previous section, we discussed the phenomenon that the result of the expert's trials showed a strong positive correlation in the area that has a smaller $t_{l}$ than that of the beginner. This would also be shown in various human physical interactions under the condition that one of the parties does not know about the dynamics and the intentions of the other. We assume that the feature of synchronising posture variations will also appear under the condition explained above.

To this end, we will attempt to apply this work's analysis to several physical interactions other than the rising-up motions (for instance, supported walking, sitting down after rising-up and interactive teaching of reaching motions). Furthermore, it will be interesting to investigate for which other types of tasks the results shown in this research also hold. 
As can be clearly seen in the demonstrated rising-up motion, physical interaction is generated from bilateral force exchange. Therefore, we will attempt to use various kinds of force sensors, floor reaction force sensors and muscle potential sensors to analyse the essential physical quantities involved in physical interaction tasks. Kuniyoshi et al. (2004) showed the existence of a knack in dynamic motions by using the essential physical quantities. In particular, we are considering the existence of the knack of PHRI in joint dynamics of a human and a robot.

\section{Conclusion}

In this paper, we investigated PHRI as an important extension of traditional HRI research. The aim of this research is to develop a humanoid robot that can work in the same space as humans and receive physical help from the latter without exposing them to any danger. A long-term goal of this research is to allow a humanoid robot to develop both motor skills and cognitive abilities in close physical interaction with a human teacher.

As a prerequisite to this goal, we have introduced a suitable control system and discussed the hardware requirements needed for secure physical human-robot interaction. We showed that flexible-joint robots are particularly well suited to this task. Furthermore, we showed that the control system can be simplified if the inherent advantages of the hardware are used. This allowed physical interaction where a human has to help a robot in its rising-up behaviour which is obviously a difficult task for traditional hardware and control systems.

We also clarified that the difference between smooth and non-smooth physical interaction can be shown using the cross-correlation value calculated from posture-change norms. Abe et al. (2003) showed the difference in motor skills between an expert and a beginner of ceramic art by focusing on the cross-correlation values of the time-series of posture data taken using a motion capture system. Our proposed evaluation method for PHRI is assumed to be an extension of their evaluation method. This difference is assumed to appear in several physical interaction tasks other than the rising-up behaviour investigated in this paper. Further research is therefore needed to investigate the general applicability of these results. The analysis results shown in this paper suggest the need to focus on the timing of the switching of desired postures to implement a motor learning system in a robot. Additionally, we assume that the results can be used for evaluating each trial, an evaluation that is necessary for a motor learning system using the physical help of a human helper. Future works will aim to provide a quantitive definition for the synchronisation between the robot and the human. We will also need to analyse whether applying weights to the joint angles in the computation of the posture change norms could improve the quality of the measure.

\section{Acknowledgment}

This paper owes much to the thoughtful and helpful comments of Prof. Minoru Asada (Osaka University). Also, special thanks are due to Mr. Heni Ben Amor (Technical University Bergakademie Freiberg) for reading the entire text in its original form.

\section{References}

Abe M, Yamamoto T, Fujinami T. 2003. A dynamical analysis of kneading using a motion capture device, Third International Workshop on Epigenetic Robotics.

Alami R, Albu-Schaeffer A, Bicchi A, Bischoff R, Chatila R, De Luca A, De Santis A, Giralt G, Guiochet J, Hirzinger G, et al. 2006. Safe and dependable physical human-robot interaction in anthropic domains: State of the art and challenges. Workshop of 2006 IEEE/RSJ International Conference on Intelligent Robots and Systems.

Asada H, Asari Y. 1988. The direct teaching of tool manipulation skills via the impedance identification of human motion, Proceedings of IEEE International Conference on Robotics and Automation, p. 1269-1274.

Kanda T, Ishiguro H, Ono T, Imai M, Nakatsu R. 2002. Development and evaluation of an interactive humanoid robot "Robovie", Proceedings of the IEEE International Conference on Robotics and Automation, p. 1848-1855.

Kosuge K, Hayashi T, Hirata Y, Tobiyama R. 2003. Dance partner robot-Ms DanceR-. Proceedings of the 2003 IEEE/RSJ International Conference on Intelligent Robots and Systems.

Kosuge K, Sato M, Kazamura N. 2000. Mobile robot helper. Proceedings of the 2000 IEEE International Conference on Robotics and Automation.

Kuniyoshi Y, Ohmura Y, Terada K, Nagakubo A, Eitoku S, Yamamoto T. 2004. Embodied basis of invariant features in execution and perception of whole-body dynamic actions. Knacks and focuses of roll-and-rise motion. Autonomous Syst. 48:189-201.

Minato T, Yoshikawa Y, Noda T, Ikemoto S, Ishiguro H, Asada M. 2007. $\mathrm{CB}^{2}$ : A child robot with biomimetic body for cognitive developmental robotics, Humanoids 2007.

Noda T, Miyashita T, Ishiguro H, Hagita N. Self-organizing superflexible skin sensors embedded on the whole, Robotics Science and System Proceedings (RSS 2008), 2008.

Suga Y, Arie H, Ogata T, Sugano S. 2004. Development of emotional communication robot, WAMOEBA-3, Proceedings of the 4th International Conference on the Advanced Mechatronics, p. 413-418. 

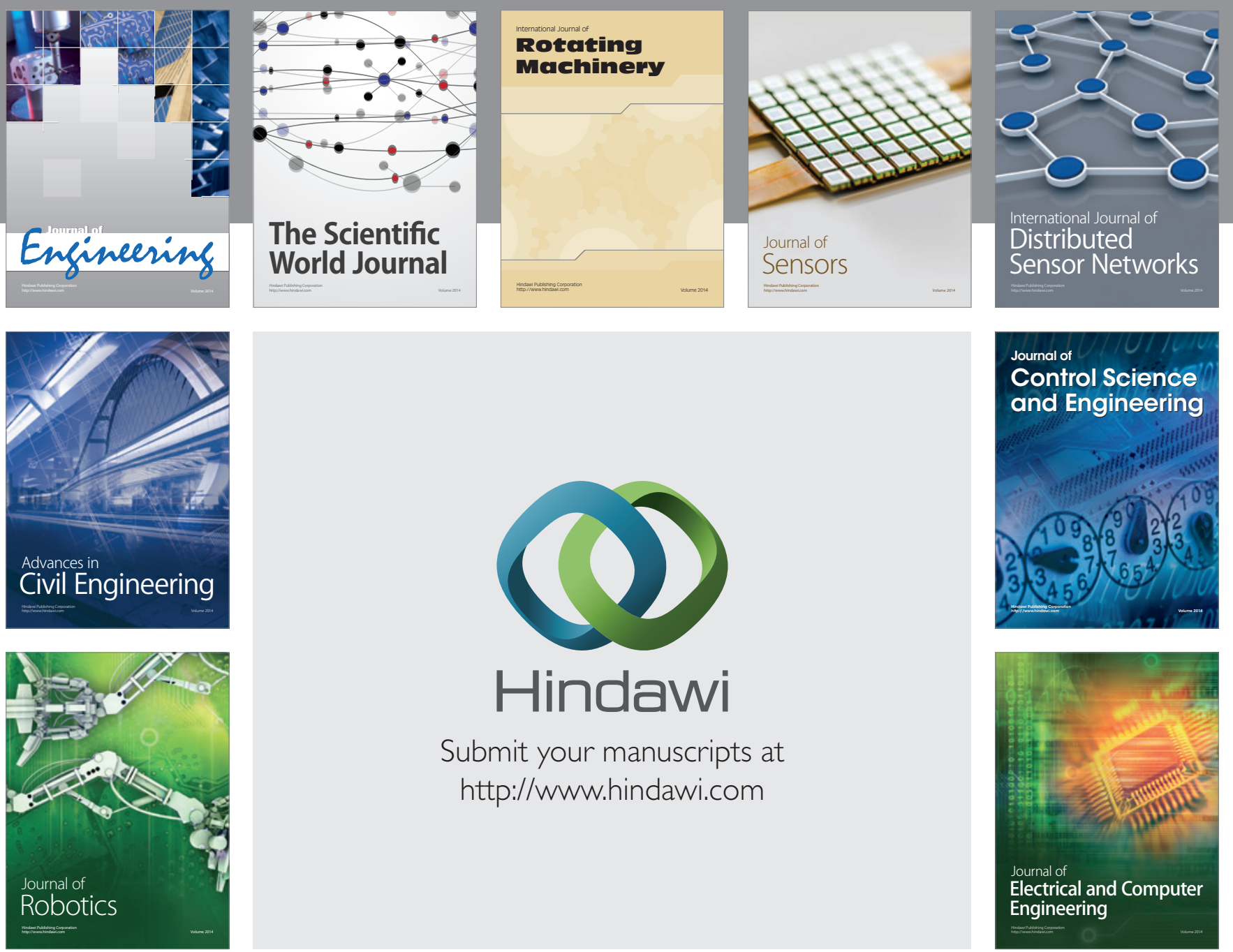

Submit your manuscripts at

http://www.hindawi.com
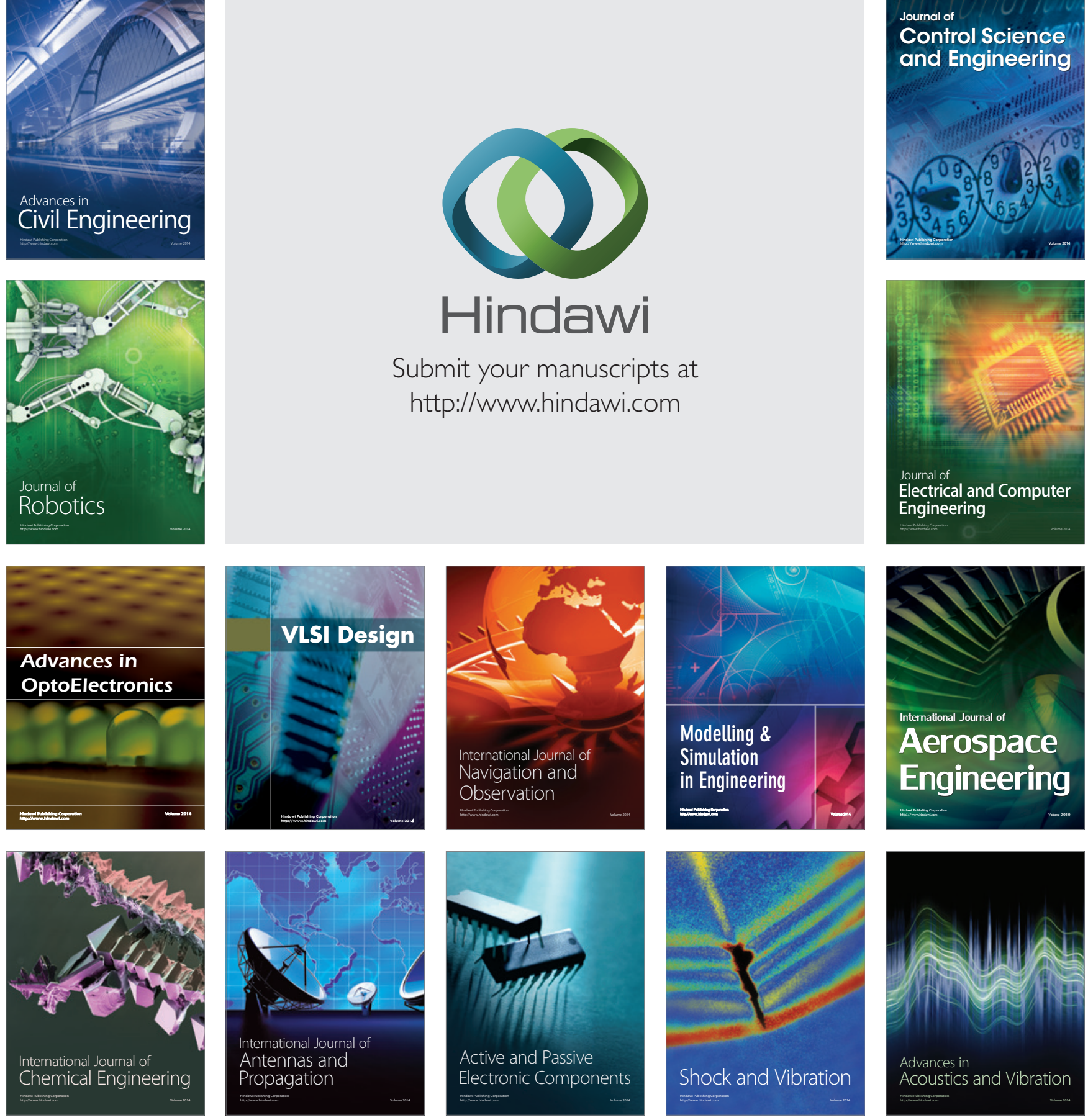